\title{
Postgraduate Corner
}

1. In quinine sulphate overdose :

a. QTc interval shortens

b. Amiodarone is the anti-arrhythmic of choice for VT with pulse.

c. Quinine induces insulin release.

d. Continuous veno-venous hemofiltration will remove quinine

2. In relation to acute aortic dissection:

a. More common in females with peak incidence between 50-70 yrs of age

b. The European Society of Cardiology classifies into type A and type B

c. Adequate beta blockade should be established before initiation of vasodilator therapy in medical management.

d. Type A aortic dissection is managed medically.

3. Regarding defibrillation true is:

a. Transthoracic impedance is approximately 100 Ohms in adults.

b. Anteroposterior electrode placement is less effective than the sterno-apical position.

c. Biphasic defibrillators have a first shock efficacy of $>86 \%$ for long duration VF

d. Biphasic defibrillators offer a survival advantatge over monophasic devices.

4. Regarding variceal bleeding false is:

a. Portal hypertension is the most common cause.

b. Terlipressin may be beneficial in reducing bleddding.

c. Broad-spectrum antibiotics should be given acutely.

d. TIPS and liver transplantation may be considered in severe cases.

5. Regarding the Sengstaken-Blakemore tube false is:

a. Effective at controlling torrential bleeding from varices.

b. It has three lumens. c. Insertion to the $55 \mathrm{~cm}$ mark at the incisors indicates that the gastric balloon position is below the gastroesophageal junction.

d. The oesophageal balloon must be deflated every 6 hours.

6. Regarding alcoholic liver disease(ALD) true is:

a. $30 \%$ of cirrhotic patients develop hepatorenal syndrome (HRS) within 1 year of diagnosis.

b. One UK unit of alcohol contains 20-24 g ethanol.

c. Ethanol metabolism causes accumulation of lipid in liver cells.

d. Infliximab is useful in preventing HRS in severe ALD.

7. Mannitol $20 \%$ :

a. $500 \mathrm{ml}$ bag contains $100 \mathrm{~g}$ of mannitol.

b. Is alkalotic.

c. Mannitol solutions may cristallise at room temperature and should be discarded.

d. $0.25-1.0 \mathrm{mg} / \mathrm{kg}$ should be given IV for raised ICP.

8. Regarding pre-eclampsia true is

a. Results from trophoblastic invasion of spiral arteries within placenta.

b. More common in primiparous women.

c. Usually presents before 20 weeks gestation.

d. Proteinuria with high BP is required for diagnosis.

9. Dominant R-wave in lead V1 on ECG is found
a. WPW type B
b. Inferior myocardial infarction
c. HOCM
d. Dressler's syndrome.

10. Regarding oesophageal Doppler true is
a. Velocity of blood $(\mathrm{m} / \mathrm{s})$ in the descending aorta can be calculated provided the aortic cross- sectional area is known.
b. It is assumed $70 \%$ of cardiac output is distributed caudally to the descending aorta.
c. Doppler probes must be removed after 1 week.
d. Is accurate when used with a working epidural.

\section{Answers}

1.c, 2.c, 3.c, 4.a, 5.d, 6.c, 7.a, 8.b, 9.c, 10.b 